\title{
Brunnian local moves of knots and Vassiliev invariants
}

\author{
by
}

Akira Yasuhara (Tokyo)

Dedicated to Professor Takao Matumoto for his 60th birthday

\begin{abstract}
K. Habiro gave a neccesary and sufficient condition for knots to have the same Vassiliev invariants in terms of $C_{k}$-moves. In this paper we give another geometric condition in terms of Brunnian local moves. The proof is simple and self-contained.
\end{abstract}

1. Introduction. We will define local moves via tangles. Our definition follows [11], [12]. A tangle $T$ is a disjoint union of properly embedded arcs in the unit 3-ball $B^{3}$. Here $T$ contains no closed arcs. A tangle $T$ is trivial if there exists a properly embedded disk in $B^{3}$ that contains $T$. A local move is a pair of trivial tangles $\left(T_{1}, T_{2}\right)$ with $\partial T_{1}=\partial T_{2}$ such that for each component $t$ of $T_{1}$ there exists a component $u$ of $T_{2}$ with $\partial t=\partial u$. Two local moves $\left(T_{1}, T_{2}\right)$ and $\left(U_{1}, U_{2}\right)$ are equivalent, denoted by $\left(T_{1}, T_{2}\right) \cong\left(U_{1}, U_{2}\right)$, if there is an orientation preserving self-homeomorphism $\psi: B^{3} \rightarrow B^{3}$ such that $\psi\left(T_{i}\right)$ and $U_{i}$ are ambient isotopic in $B^{3}$ relative to $\partial B^{3}$ for $i=1,2$. A local move $\left(T_{1}, T_{2}\right)$ is trivial if $\left(T_{1}, T_{2}\right)$ is equivalent to the local move $\left(T_{1}, T_{1}\right)$. Note that $\left(T_{1}, T_{2}\right)$ is trivial if and only if $T_{1}$ and $T_{2}$ are ambient isotopic in $B^{3}$ relative to $\partial B^{3}$.

Let $\left(T_{1}, T_{2}\right)$ be a local move, and let $t_{1}, \ldots, t_{k}$ and $u_{1}, \ldots, u_{k}$ be the components of $T_{1}$ and $T_{2}$ respectively with $\partial t_{i}=\partial u_{i}(i=1, \ldots, k)$. We call $\left(T_{1}, T_{2}\right)$ a $k$-component Brunnian local move $(k \geq 2)$, or $B_{k}$-move, if each local move $\left(T_{1}-t_{i}, T_{2}-u_{i}\right)$ is trivial $(i=1, \ldots, k)$ [10]. If $\left(T_{1}, T_{2}\right)$ is Brunnian, then $\left(T_{2}, T_{1}\right)$ is also Brunnian. For example, a crossing change is a $B_{2}$-move, the delta-move defined in [7] is a $B_{3}$-move, and a $C_{k}$-move defined in [3], [4] is a $B_{k+1}$-move.

2000 Mathematics Subject Classification: Primary 57M25.

Key words and phrases: Brunnian move, Vassiliev invariant, band sum. 
Let $K_{1}$ and $K_{2}$ be oriented knots in the three-sphere $S^{3}$ with a fixed orientation. We say that $K_{2}$ is obtained from $K_{1}$ by a local move $\left(T_{1}, T_{2}\right)$ if there is an orientation preserving embedding $h: B^{3} \rightarrow S^{3}$ such that $\left(h^{-1}\left(K_{1}\right), h^{-1}\left(K_{2}\right)\right) \cong\left(T_{1}, T_{2}\right)$ and $K_{1}-h\left(B^{3}\right)=K_{2}-h\left(B^{3}\right)$ as oriented tangles. Two oriented knots $K_{1}$ and $K_{2}$ are $B_{k}$-equivalent if $K_{2}$ is obtained from $K_{1}$ by a finite sequence of $B_{k}$-moves and ambient isotopies. This relation is an equivalence relation on knots.

We have the following geometric condition for knots to have the same value of Vassiliev invariant.

Theorem 1 (cf. Goussarov-Habiro Theorem [4], [2]). Two knots $K_{1}$ and $K_{2}$ are $B_{l+1}$-equivalent if and only if their values of any Vassiliev invariant of order $\leq l-1$ are equal.

REMARK. The authors of [5] and [6] showed independently that $B_{l+1^{-}}$ and $C_{l}$-equivalence classes coincide. Therefore, the theorem above and the Goussarov-Habiro Theorem are consequences of each other. Although Theorem 1 can be obtained as a corollary of the Goussarov-Habiro Theorem the author believes that a new and self-contained proof is worth presenting. Moreover, the arguments used in our proof are shorter and simpler compared to those given in [4], [2] and [12] for the proof of the Goussarov-Habiro Theorem.

Let $l$ be a positive integer and let $k_{1}, \ldots, k_{l}(\geq 2)$ be integers. Suppose that for each $P \subset\{1, \ldots, l\}$ we have an oriented knot $K_{P}$ in $S^{3}$ and there are orientation preserving embeddings $h_{i}: B^{3} \rightarrow S^{3}(i=1, \ldots, l)$ such that:

(1) $h_{i}\left(B^{3}\right) \cap h_{j}\left(B^{3}\right)=\emptyset$ if $i \neq j$,

(2) $K_{P}-\bigcup_{i=1}^{l} h_{i}\left(B^{3}\right)=K_{P^{\prime}}-\bigcup_{i=1}^{l} h_{i}\left(B^{3}\right)$ for all $P, P^{\prime} \subset\{1, \ldots, l\}$,

(3) $\left(h_{i}^{-1}\left(K_{\emptyset}\right), h_{i}^{-1}\left(K_{\{1, \ldots, l\}}\right)\right)$ is a $B_{k_{i}}$-move $(i=1, \ldots, l)$, and

(4) $K_{P} \cap h_{i}\left(B^{3}\right)= \begin{cases}K_{\{1, \ldots, l\}} \cap h_{i}\left(B^{3}\right) & \text { if } i \in P, \\ K_{\emptyset} \cap h_{i}\left(B^{3}\right) & \text { otherwise. }\end{cases}$

Then we call the set $\left\{K_{P} \mid P \subset\{1, \ldots, l\}\right\}$ of oriented knots a singular knot of type $B\left(k_{1}, \ldots, k_{l}\right)$. Let $\mathcal{K}$ be the set of knots, $A$ an abelian group, and $\varphi: \mathcal{K} \rightarrow A$ an invariant. We say that $\varphi$ is a finite type invariant of type $B\left(k_{1}, \ldots, k_{l}\right)$ if for any singular knot $\left\{K_{P} \mid P \subset\{1, \ldots, l\}\right\}$ of type $B\left(k_{1}, \ldots, k_{l}\right)$,

$$
\sum_{P \subset\{1, \ldots, l\}}(-1)^{|P|} \varphi\left(K_{P}\right)=0 .
$$

Since a $B_{2}$-move is realized by some crossing changes we see that an invariant $\varphi: \mathcal{K} \rightarrow A$ is a finite type invariant of type $B(\underbrace{2, \ldots, 2}_{l})$ if and only if it is a
Vassiliev invariant of order $\leq l-1$.

In order to prove Theorem 1, we need the following theorems. 
TheOREM 2 (cf. [4, Theorem 5.4]). The set of $B_{k}$-equivalence classes, denoted by $\mathcal{K} / B_{k}$, of oriented knots in $S^{3}$ forms an abelian group under connected sum of oriented knots.

Theorem 3 (cf. [12, Theorem 1.2]). Let $l(\geq 2)$ and $k_{1}, \ldots, k_{l}(\geq 2)$ be integers, and $k-1=\left(k_{1}-1\right)+\cdots+\left(k_{l}-1\right)$. Then the projection $p_{k}: \mathcal{K} \rightarrow \mathcal{K} / B_{k}$ is a finite type invariant of type $B\left(k_{1}, \ldots, k_{l}\right)$.

Remark. Since a $C_{k}$-move is the same as a $B_{k+1}$-move, Theorem 2 follows from [4, Theorem 5.4]. Theorem 3 is similar to [12, Theorem 1.2]. In order to give a self-contained proof of Theorem 1, we will give self-contained proofs of Theorems 2 and 3. Although the reasonings given in the proofs of Theorems 2 and 3 are analogous to those in [4] (and also in [11] and [12]) we provide simpler and shorter arguments.

2. Band description. It is known that any knot can be expressed as a "band sum" of the trivial knot and a split union of some Hopf links [8], [13] (or Borromean rings [14]). K. Taniyama and the author showed that if two knots are $C_{k}$-equivalent, then one can be expressed as a band sum of the other and a split union of certain $(k+1)$-component Brunnian links [11], [12]. By similar arguments to those in [11], we describe a relation between $B_{k}$-equivalence and a certain band sum.

Let $\left(T_{1}, T_{2}\right)$ be a $k$-component Brunnian local move. Let $T \subset B^{3}$ be the trivial $k$-string tangle illustrated in Figure 1 , and let $D$ be the disjoint union of the $k$ disks bounded by $T$ and arcs in $\partial B^{3}$ (see Figure 2). Since $T_{2}$ is a trivial tangle, there is a tangle $S$ such that $(S, T)$ and $\left(T_{1}, T_{2}\right)$ are equivalent. Then the pair $(S, \partial D-T)$ is called a $B_{k}$-link model (see Figure 3 ).

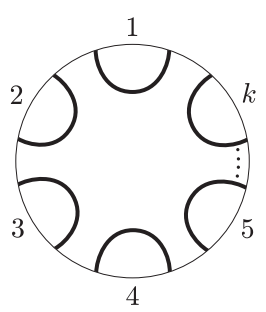

Fig. 1

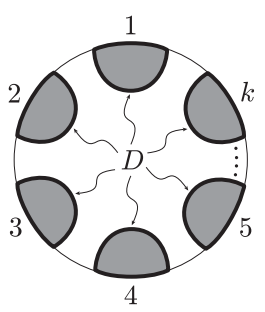

Fig. 2

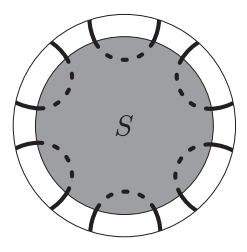

$B_{k}$-move

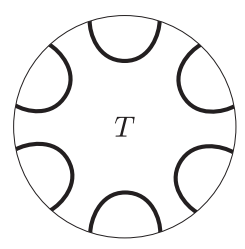

Fig. 3

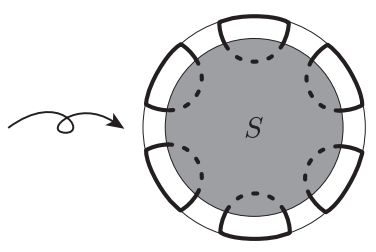

$B_{k}$-link model 
Let $\left(\alpha_{i}, \beta_{i}\right)$ be $B_{\varrho(i)}$-link models $(i=1, \ldots, l)$, and $K$ an oriented knot (respectively a tangle). Let $\psi_{i}: B^{3} \rightarrow S^{3}$ (respectively $\psi_{i}: B^{3} \rightarrow \operatorname{int} B^{3}$ ) be an orientation preserving embedding for $i=1, \ldots, l$, and let $b_{1,1}, \ldots, b_{1, \varrho(1)}$, $b_{2,1}, \ldots, b_{2, \varrho(2)}, \ldots, b_{l, 1}, \ldots, b_{l, \varrho(l)}$ be mutually disjoint disks embedded in $S^{3}$ (respectively $B^{3}$ ). Suppose that they satisfy the following conditions;

(1) $\psi_{i}\left(B^{3}\right) \cap \psi_{j}\left(B^{3}\right)=\emptyset$ if $i \neq j$,

(2) $\psi_{i}\left(B^{3}\right) \cap K=\emptyset$ for each $i$,

(3) $b_{i, k} \cap K=\partial b_{i, k} \cap K$ is an arc for each $i, k$,

(4) $b_{i, k} \cap \bigcup_{j=1}^{l} \psi_{j}\left(B^{3}\right)=\partial b_{i, k} \cap \psi_{i}\left(B^{3}\right)$ is a component of $\psi_{i}\left(\beta_{i}\right)$ for each $i, k$.

Let $J$ be an oriented knot (respectively a tangle) defined by

$$
J=K \cup\left(\bigcup_{i, k} \partial b_{i, k}\right) \cup\left(\bigcup_{i=1}^{l} \psi_{i}\left(\alpha_{i}\right)\right)-\bigcup_{i, k} \operatorname{int}\left(\partial b_{i, k} \cap K\right)-\bigcup_{i=1}^{l} \psi_{i}\left(\operatorname{int} \beta_{i}\right),
$$

where the orientation of $J$ coincides with that of $K$ on $K-\bigcup_{i, k} b_{i, k}$ if $K$ is oriented. We call each $b_{i, k}$ a band. Each image $\psi_{i}\left(B^{3}\right)$ is called a link ball. We set $\mathcal{B}_{i}=\left(\left(\alpha_{i}, \beta_{i}\right), \psi_{i},\left\{b_{i, 1}, \ldots, b_{i, \varrho(i)}\right\}\right)$ and call $\mathcal{B}_{i}$ a $B_{\varrho(i)}$-chord. We denote $J$ by $J=\Omega\left(K ;\left\{\mathcal{B}_{1}, \ldots, \mathcal{B}_{l}\right\}\right)$, and say that $J$ is a band sum of $K$ and chords $\mathcal{B}_{1}, \ldots, \mathcal{B}_{l}$, or a band sum of $K$ and $\left\{\mathcal{B}_{1}, \ldots, \mathcal{B}_{l}\right\}$.

From now on we consider knots up to ambient isotopy of $S^{3}$ and tangles up to ambient isotopy of $B^{3}$ relative to $\partial B^{3}$ without explicit mention.

By the definitions of a $B_{k}$-move and a $B_{k}$-link model, we have:

Sublemma 4 (cf. [12, Sublemmas 3.3 and 3.5]).

(1) A local move $\left(T_{1}, T_{2}\right)$ is a $B_{k}$-move if and only if $T_{1}$ is a band sum of $T_{2}$ and a $B_{k}$-link model.

(2) $A$ knot $J$ is obtained from a knot $K$ by a single $B_{k}$-move if and only if $K$ is a band sum of $J$ and $a B_{k}$-link model.

Note that, by Sublemma 4(1), a set $\mathbf{K}$ of knots is a singular knot of type $B\left(k_{1}, \ldots, k_{l}\right)$ if and only if there is a knot $K$ and a band sum $J=$ $\Omega\left(K ;\left\{\mathcal{B}_{1}, \ldots, \mathcal{B}_{l}\right\}\right)$ of $K$ and $B_{k_{i}}$-chords $\mathcal{B}_{i}(i=1, \ldots, l)$ such that

$$
\mathbf{K}=\left\{\Omega\left(K ; \bigcup_{i \in P}\left\{\mathcal{B}_{i}\right\}\right) \mid P \subset\{1, \ldots, l\}\right\} .
$$

Sublemma 5 (cf. [12, Sublemma 3.5]). Let $K, J$ and $I$ be oriented knots (or tangles). Suppose that $J=\Omega\left(K ;\left\{\mathcal{B}_{1}, \ldots, \mathcal{B}_{l}\right\}\right)$ for some chords $\mathcal{B}_{1}, \ldots, \mathcal{B}_{l}$ and $I=\Omega(J ;\{\mathcal{B}\})$ for some $B_{k}$-chord $\mathcal{B}$. Then there is a $B_{k}$-chord $\mathcal{B}^{\prime}$ such that $I=\Omega\left(K ;\left\{\mathcal{B}_{1}, \ldots, \mathcal{B}_{l}, \mathcal{B}^{\prime}\right\}\right)$. Moreover, if for a subset $P$ of $\{1, \ldots, l\}$ the link ball or the bands of $\mathcal{B}$ intersect either the link ball or the bands of $\mathcal{B}_{i}$ only when $i \in P$, then $\Omega\left(\Omega\left(K ; \bigcup_{i \in P}\left\{\mathcal{B}_{i}\right\}\right) ;\{\mathcal{B}\}\right)=\Omega\left(K ;\left(\bigcup_{i \in P}\left\{\mathcal{B}_{i}\right\}\right) \cup\left\{\mathcal{B}^{\prime}\right\}\right)$. 
Proof. If the bands and the link ball of $\mathcal{B}$ are disjoint from those of $\mathcal{B}_{1}, \ldots, \mathcal{B}_{l}$ then $I=\Omega\left(K ;\left\{\mathcal{B}_{1}, \ldots, \mathcal{B}_{l}, \mathcal{B}\right\}\right)$. If not, then we deform $I$ up to ambient isotopy as follows. By thinning and shrinking the bands and the link ball of $\mathcal{B}$ respectively, we may assume that the link ball of $\mathcal{B}$ intersects neither the bands nor the link balls of $\mathcal{B}_{1}, \ldots, \mathcal{B}_{l}$. And by sliding the bands of $\mathcal{B}$ along $J$, we may also assume that the intersection of the bands with $J$ is disjoint from the bands and the link balls of $\mathcal{B}_{1}, \ldots, \mathcal{B}_{l}$. Then we sweep the bands of $\mathcal{B}$ out of the link balls of $\mathcal{B}_{1}, \ldots, \mathcal{B}_{l}$. Note that this is always possible since the tangles of a local move are trivial. Finally, we sweep the intersection of the bands of $\mathcal{B}$ and the bands of $\mathcal{B}_{1}, \ldots, \mathcal{B}_{l}$ out of the intersection of the bands of $\mathcal{B}_{1}, \ldots, \mathcal{B}_{l}$ and $K$. Let $\mathcal{B}^{\prime}$ be the result of the deformation of $\mathcal{B}$ described above. Then it is not hard to see that $\mathcal{B}^{\prime}$ is the desired chord.

By repeated applications of Sublemmas 4 and 5 we immediately have the following lemma.

Lemma 6 (cf. [12, Lemma 3.6]). Let $k$ be a positive integer and let $K$ and $J$ be oriented knots (or tangles). Then $K$ and $J$ are $B_{k}$-equivalent if and only if $J$ is a band sum of $K$ and some $B_{k}$-link models.

Since the local moves illustrated in Figures 4 and 5 are a $B_{k+1}$-move and $B_{j+k-1}$-move respectively, the following two lemmas follow from Sublemma 5 .

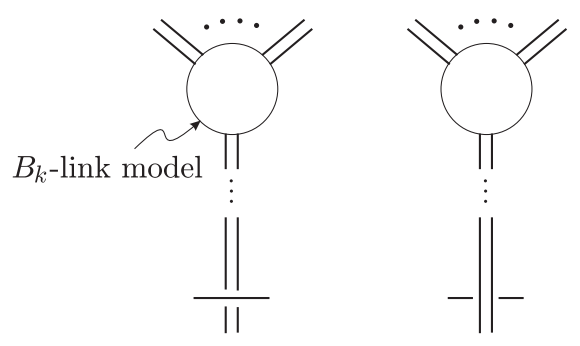

Fig. 4
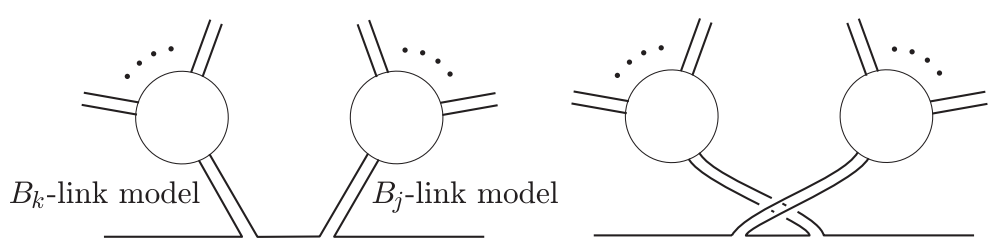

Fig. 5

LEMMA 7 (cf. [12, Lemma 3.8]). Let $K, J=\Omega\left(K ;\left\{\mathcal{B}_{1}, \ldots, \mathcal{B}_{l}, \mathcal{B}_{0}\right\}\right)$ and $I=\Omega\left(K ;\left\{\mathcal{B}_{1}, \ldots, \mathcal{B}_{l}, \mathcal{B}_{0}^{\prime}\right\}\right)$ be oriented knots, where $\mathcal{B}_{1}, \ldots, \mathcal{B}_{l}$ are chords and $\mathcal{B}_{0}, \mathcal{B}_{0}^{\prime}$ are $B_{k}$-chords. Suppose that $J$ and $I$ differ locally as illustrated in Figure 4, i.e., $I$ is obtained from $J$ by a crossing change between $K$ and a band 
of $\mathcal{B}_{0}$. Then $I$ is obtained from $J$ by a $B_{k+1}$-move. Moreover, there is a $B_{k+1^{-}}$chord $\mathcal{B}$ such that $\Omega\left(K ;\left(\bigcup_{i \in P}\left\{\mathcal{B}_{i}\right\}\right) \cup\left\{\mathcal{B}_{0}\right\}\right)=\Omega\left(K ;\left(\bigcup_{i \in P}\left\{\mathcal{B}_{i}\right\}\right) \cup\left\{\mathcal{B}_{0}^{\prime}, \mathcal{B}\right\}\right)$ for any subset $P$ of $\{1, \ldots, l\}$.

Lemma 8 (cf. [12, Lemma 3.9]). Let $K, J=\Omega\left(K ;\left\{\mathcal{B}_{1}, \ldots, \mathcal{B}_{l}, \mathcal{B}_{0 j}, \mathcal{B}_{0 k}\right\}\right)$ and $I=\Omega\left(K ;\left\{\mathcal{B}_{1}, \ldots, \mathcal{B}_{l}, \mathcal{B}_{0 j}^{\prime}, \mathcal{B}_{0 k}^{\prime}\right\}\right)$ be oriented knots, where $\mathcal{B}_{1}, \ldots, \mathcal{B}_{l}$ are chords and $\mathcal{B}_{0 j}, \mathcal{B}_{0 j}^{\prime}$ (respectively $\mathcal{B}_{0 k}, \mathcal{B}_{0 k}^{\prime}$ ) are $B_{j}$-chords (respectively $B_{k}$ chords). Suppose that $J$ and $I$ differ locally as illustrated in Figure 5. Then $I$ is obtained from $J$ by a $B_{j+k-1}$-move. Moreover, there is a $B_{j+k-1}$-chord $\mathcal{B}$ such that $\Omega\left(K ;\left(\bigcup_{i \in P}\left\{\mathcal{B}_{i}\right\}\right) \cup\left\{\mathcal{B}_{0 j}, \mathcal{B}_{0 k}\right\}\right)=\Omega\left(K ;\left(\bigcup_{i \in P}\left\{\mathcal{B}_{i}\right\}\right) \cup\left\{\mathcal{B}_{0 j}^{\prime}, \mathcal{B}_{0 k}^{\prime}, \mathcal{B}\right\}\right)$ for any subset $P$ of $\{1, \ldots, l\}$.

We call the change from $J$ to $I$ in Lemma 8 a band exchange.

For a $C_{k}$-move, "band description" is also defined, and Sublemmas 4,5 , Lemmas 6, 7 and 8 hold [12]. However, the proofs given in [12] are not as obvious as ours. In fact, more complicated arguments are needed. In contrast, we need some arguments to prove the following lemma, which is trivial for a $C_{k}$-move.

Lemma 9. Let $\left(T_{1}, T_{2}\right)$ be a $B_{k}$-move. For any integer $l(\leq k), T_{2}$ is obtained from $T_{1}$ by $B_{l}$-moves. In particular, $B_{k}$-equivalent knots are $B_{l}$ equivalent.

Proof. Let $t_{1}, \ldots, t_{k}$ and $u_{1}, \ldots, u_{k}$ be the components with $\partial t_{i}=\partial u_{i}$ $(i=1, \ldots, k)$ of $T_{1}$ and $T_{2}$ respectively. We may assume that $\left(T_{1}, T_{2}\right)$ has a diagram in the unit disk such that $T_{1}-t_{1}$ and $T_{2}$ have no crossings.

Since $\left(T_{1}-t_{2}, T_{2}-u_{2}\right)$ is a trivial local move, $T_{2}$ is obtained from $T_{1}$ by $B_{2}$-moves that correspond to crossing changes between $t_{1}$ and $t_{2}$. By Lemma $6, T_{1}$ is a band sum, $\Omega\left(T_{2} ; \mathbf{B}_{2}\right)$, of $T_{2}$ and a set $\mathbf{B}_{2}$ of $B_{2}$-chords. Note that no band of $B_{2}$-chords intersects $T_{2}-\left(u_{1} \cup u_{2}\right)$.

Since $\left(\Omega\left(T_{2} ; \mathbf{B}_{2}\right)-t_{3}, T_{2}-u_{3}\right)=\left(T_{1}-t_{3}, T_{2}-u_{3}\right)$ is a trivial local move, $T_{2}$ is obtained from $T_{1}$ by $B_{3}$-moves that correspond to crossing changes between $t_{3}$ and some bands of $B_{2}$-chords. By Lemma $6, T_{1}$ is a band sum $\Omega\left(T_{2} ; \mathbf{B}_{3}\right)$ of $T_{2}$ and a set $\mathbf{B}_{3}$ of $B_{3}$-chords. Note that no bands of $B_{3}$-chords intersects $T_{2}-\left(u_{1} \cup u_{2} \cup u_{3}\right)$.

Continuing this process we obtain the conclusion.

\section{Proofs of Theorems 1, 2 and 3}

Proof of Theorem 3. Let $K_{0}$ be a knot and $K_{1}$ a band sum of $K_{0}$ and $B_{k_{j}}$-chords $\mathcal{B}_{k_{j}, j}(j=1, \ldots, l)$. It is sufficient to show that

$$
\sum_{P \subset\{1, \ldots, l\}}(-1)^{|P|}\left[\Omega\left(K_{0} ; \bigcup_{j \in P}\left\{\mathcal{B}_{k_{j}, j}\right\}\right)\right]=0 \in \mathcal{K} / B_{k},
$$

where $[K]$ is the $B_{k}$-equivalence class which contains the knot $K$. 
Set

$$
K_{P}=\Omega\left(K_{0} ; \bigcup_{j \in P}\left\{\mathcal{B}_{k_{j}, j}\right\}\right) .
$$

ClaIm. The knot $K_{1}\left(=K_{\{1, \ldots, l\}}\right)$ is $B_{k}$-equivalent to a band sum of $K_{0}$ $\left(=K_{\emptyset}\right)$ and a set $\bigcup_{i, j}\left\{\mathcal{B}_{i, j}\right\}$ of local chords such that

(1) $\mathcal{B}_{i, j}$ is a $B_{i}$-chord $(i<k)$ and it has an associated subset $\omega\left(\mathcal{B}_{i, j}\right) \subset$ $\{1, \ldots, l\}$ with $\sum_{t \in \omega\left(\mathcal{B}_{i, j}\right)}\left(k_{t}-1\right) \leq i-1$,

(2) for each $P \subset\{1, \ldots, l\}$,

$$
\left[K_{P}\right]=\left[\Omega\left(K_{0} ; \bigcup_{\omega\left(\mathcal{B}_{i, j}\right) \subset P}\left\{\mathcal{B}_{i, j}\right\}\right)\right]
$$

Here a chord $\mathcal{B}_{i, j}$ is called a local chord if there is a 3 -ball $B$ such that $B$ contains all the bands and the link ball of $\mathcal{B}_{i, j}, B$ does not intersect any other bands or link balls, and $\left(B, B \cap K_{0}\right)$ is a trivial ball-arc pair.

Before proving the Claim, we will finish the proof of Theorem 3. Suppose $K_{1}$ is $B_{k^{-}}$equivalent to a band sum of $K_{0}$ and some local chords $\mathcal{B}_{i, j}$. Each $\mathcal{B}_{i, j}$ represents a knot $K_{i, j}$ which is connected summed with $K_{0}$. So the band sum is a connected sum of $K_{0}$ and $K_{i, j}$ 's. Then we have

$$
\begin{aligned}
\sum_{P \subset\{1, \ldots, l\}}(-1)^{|P|}\left[\Omega\left(K_{0} ; \bigcup_{\omega\left(\mathcal{B}_{i, j}\right) \subset P}\left\{\mathcal{B}_{i, j}\right\}\right)\right] \\
=\sum_{P \subset\{1, \ldots, l\}}(-1)^{|P|}\left(\left[K_{0}\right]+\sum_{\omega\left(\mathcal{B}_{i, j}\right) \subset P}\left[K_{i, j}\right]\right) \\
=\sum_{P \subset\{1, \ldots, l\}}(-1)^{|P|}\left[K_{0}\right]+\sum_{P \subset\{1, \ldots, l\}}(-1)^{|P|}\left(\sum_{\omega\left(\mathcal{B}_{i, j}\right) \subset P}\left[K_{i, j}\right]\right) \\
=0+\sum_{i, j}\left(\sum_{P \subset\{1, \ldots, l\}, \omega\left(\mathcal{B}_{i, j}\right) \subset P}(-1)^{|P|}\right)\left[K_{i, j}\right] .
\end{aligned}
$$

We consider the coefficient of $\left[K_{i, j}\right]$. Since $\sum_{t \in \omega\left(\mathcal{B}_{i, j}\right)}\left(k_{t}-1\right)<k-1, \omega\left(\mathcal{B}_{i, j}\right)$ is a proper subset of $\{1, \ldots, l\}$. We may assume that $\omega\left(\mathcal{B}_{i, j}\right)$ does not contain $a \in\{1, \ldots, l\}$. Then

$$
\begin{aligned}
& \sum_{P \subset\{1, \ldots, l\}, \omega\left(\mathcal{B}_{i, j}\right) \subset P}(-1)^{|P|}=\sum_{P \subset\{1, \ldots, l\} \backslash\{a\}, \omega\left(\mathcal{B}_{i, j}\right) \subset P}(-1)^{|P|} \\
& +\sum_{P \subset\{1, \ldots, l\} \backslash\{a\}, \omega\left(\mathcal{B}_{i, j}\right) \subset P}(-1)^{|P \cup\{a\}|}=0 .
\end{aligned}
$$

Thus, we have the conclusion.

Now we will show the Claim. 
Proof of Claim. We first set $\omega\left(\mathcal{B}_{k_{j}, j}\right)=\{j\}$ for $j=1, \ldots, l$. Then we have $\sum_{t \in \omega\left(\mathcal{B}_{k_{j}, j}\right)}\left(k_{t}-1\right)=k_{j}-1<k-1$ and

$$
K_{P}=\Omega\left(K_{0} ; \bigcup_{\omega\left(\mathcal{B}_{k_{j}, j}\right) \subset P}\left\{\mathcal{B}_{k_{j}, j}\right\}\right)
$$

Note that a crossing change between bands can be realized by crossing changes between $K_{0}$ and a band as illustrated in Figure 6. Therefore we can deform each chord into a local chord by (i) crossing changes between $K_{0}$ and bands, and (ii) band exchanges.

(i) When we perform a crossing change between $K_{0}$ and a $B_{p}$-band of a $B_{p^{-}}$chord $\mathcal{B}_{p, q}$ with $p \leq k-2$, by using Lemma 7 , we introduce a new $B_{p+1^{-}}$ chord $\mathcal{B}_{p+1, r}$ and we set $\omega\left(\mathcal{B}_{p+1, r}\right)=\omega\left(\mathcal{B}_{p, q}\right)$ so that conditions (1) and (2) still hold. By Lemma 7 , a crossing change between $K_{0}$ and a $B_{k-1}$-band is realized by a $B_{k}$-move and therefore does not change the $B_{k}$-equivalence class.

(ii) If we perform a band exchange between a $B_{p^{-}}$chord $\mathcal{B}_{p, q}$ and a $B_{r^{-}}$ chord $\mathcal{B}_{r, s}$ with $p+r \leq k$, then, by using Lemma 8, we introduce a new $B_{p+r-1}$-chord $\mathcal{B}_{p+r-1, n}$ and set $\omega\left(\mathcal{B}_{p+r-1, n}\right)=\omega\left(\mathcal{B}_{p, q}\right) \cup \omega\left(\mathcal{B}_{r, s}\right)$ so that conditions (1) and (2) still hold. By Lemmas 8 and 9, a band exchange between a $B_{p}$-chord $\mathcal{B}_{p, q}$ and a $B_{r}$-chord $\mathcal{B}_{r, s}$ with $p+r \geq k+1$ does not change the $B_{k}$-equivalence class.

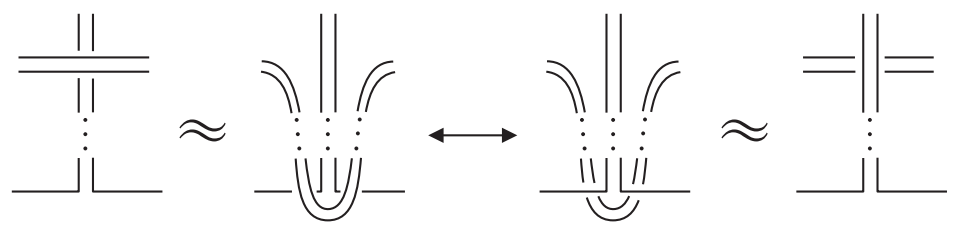

Fig. 6

Proof of Theorem 2. It is sufficient to show the existence of an inverse element for a given knot $K$. Suppose that there is a knot $J$ such that $K \# J$ is $B_{k}$-equivalent to a trivial knot $O$. Then, by Lemma $6, O$ is a band sum of $K \# J$ and some $B_{k}$-chords. By using Lemma 7 , we deform $O$ up to $B_{k+1}$-equivalence so that the $B_{k}$-chords are local chords. Then the result is a connected sum of $K \# J$ and some knots $K_{1}, \ldots, K_{n}$ that correspond to local chords. Hence $K \# J \# K_{1} \# \cdots \# K_{n}$ is $B_{k+1^{-}}$equivalent to $O$. Thus $J \# K_{1} \# \cdots \# K_{n}$ is the desired knot.

Proof of Theorem 1. It is not hard to see that $B_{l+1}$-equivalent knots are also $l$-similar [9] $((l-1)$-equivalent [1]).

By Theorem 3, the projection $p_{l+1}: \mathcal{K} \rightarrow \mathcal{K} / B_{l+1}$ is a Vassiliev invariant of order $\leq l-1$. If two knots have the same values of any Vassiliev invariant of order $\leq l-1$, then they are $B_{l+1}$-equivalent. 
Acknowledgements. The author would like to thank the referee for his/her useful comments.

\section{References}

[1] M. Gusarov, On n-equivalence of knots and invariants of finite degree, in: O. Viro (ed.), Topology of Manifolds and Varieties, Providence, RI, Amer. Math. Soc., 1994, 173-192.

[2] - Variations of knotted graphs. The geometric technique of n-equivalence, Algebra i Analiz 12 (2000), 79-125 (in Russian); English transl.: St. Petersburg Math. J. 12 (2001), 569-604.

[3] K. Habiro, Aru musubime no kyokusyo sousa no zoku ni tuite, Master thesis, Tokyo Univ., 1994 (in Japanese).

[4] -, Claspers and finite type invariants of links, Geom. Topol. 4 (2000), 1-83.

[5] -, Brunnian links, claspers and Goussarov-Vassiliev finite type invariants, preprint.

[6] H. A. Miyazawa and A. Yasuhara, Classification of n-component Brunnian links up to $C_{n}$-move, Topology Appl., to appear.

[7] H. Murakami and Y. Nakanishi, On a certain move generating link-homology, Math. Ann. 284 (1989), 75-89.

[8] S. Suzuki, Local knots of 2-spheres in 4-manifolds, Proc. Japan Acad. 45 (1969), $34-38$.

[9] K. Taniyama, On similarity of links, Gakujutsu Kenkyu, School of Education, Waseda University, Series of Matematics, 41 (1993), 33-36.

[10] K. Taniyama and A. Yasuhara, Realization of knots and links in a spatial graph, Topology Appl. 112 (2001), 87-109.

[11] - - - Local moves on spatial graphs and finite type invariants, Pacific J. Math. 211 (2003), 183-200.

[12] -, -, Band description of knots and Vassiliev invariants, Math. Proc. Cambridge Philos. Soc. 133 (2002), 325-343.

[13] M. Yamamoto, Knots in spatial embeddings of the complete graph on four vertices, Topology Appl. 36 (1990), 291-298.

[14] A. Yasuhara, Delta-unknotting operation and adaptability of certain graphs, in: Knots '96 (Tokyo), S. Suzuki (ed.), World Sci., 1997, 115-121.

Department of Mathematics

Tokyo Gakugei University

Nukuikita 4-1-1, Koganei, Tokyo 184-8501, Japan

E-mail: yasuhara@u-gakugei.ac.jp

Received 26 March 2005;

in revised form 22 January 2006 